Research Paper

\title{
Risk Factors of Intraoperative Dysglycemia in Elderly Surgical Patients
}

\author{
Cornelia Knaak ${ }^{1}$, Tobias Wollersheim ${ }^{1,2}$, Rudolf Mörgeli ${ }^{1}$, Claudia Spies ${ }^{\circledR}{ }^{\bowtie}$, Gerald Vorderwülbecke ${ }^{1}$, \\ Victoria Windmann ${ }^{1}$, Sophia Kuenz ${ }^{1}$, Maryam Kurpanik ${ }^{1}$, Gunnar Lachmann ${ }^{1,2}$; on behalf of the BIOCOG \\ Consortium
}

1. Department of Anesthesiology and Operative Intensive Care Medicine (CCM, CVK), Charité - Universitätsmedizin Berlin, corporate member of Freie Universität Berlin, Humboldt-Universität zu Berlin, and Berlin Institute of Health, Augustenburger Platz 1, D-13353 Berlin, Germany

2. Berlin Institute of Health (BIH), Anna-Louisa-Karsch-Str. 2, D-10178 Berlin, Germany

$\triangle$ Corresponding author: Prof. Dr. Claudia Spies, Department of Anesthesiology and Operative Intensive Care Medicine (CCM, CVK), Charité Universitätsmedizin Berlin, corporate member of Freie Universität Berlin, Humboldt-Universität zu Berlin, and Berlin Institute of Health, Augustenburger Platz 1, D-13353 Berlin, Germany. Phone: +49 30450551102

(c) Ivyspring International Publisher. This is an open access article distributed under the terms of the Creative Commons Attribution (CC BY-NC) license (https://creativecommons.org/licenses/by-nc/4.0/). See http://ivyspring.com/terms for full terms and conditions.

Received: 2019.01.09; Accepted: 2019.03.23; Published: 2019.05.10

\begin{abstract}
BACKGROUND: Dysglycemia is associated with adverse outcome including increased morbidity and mortality in surgical patients. Acute insulin resistance due to the surgical stress response is seen as a major cause of so-called stress hyperglycemia. However, understanding of factors determining blood glucose (BG) during surgery is limited. Therefore, we investigated risk factors contributing to intraoperative dysglycemia.

METHODS: In this subgroup investigation of the BIOCOG study, we analyzed 87 patients of $\geq 65$ years with tight intraoperative BG measurement every 20 min during elective surgery. Dysglycemia was defined as at least one intraoperative BG measurement outside the recommended target range of $80-150 \mathrm{mg} / \mathrm{dL}$. Additionally, all postoperative BG measurements in the ICU were obtained. Multivariable logistic regression analysis adjusted for age, sex, American Society of Anesthesiologists (ASA) status, diabetes, type and duration of surgery, minimum Hemoglobin $(\mathrm{Hb})$ and mean intraoperative norepinephrine use was performed to identify risk factors of intraoperative dysglycemia.

RESULTS: 46 (52.9\%) out of 87 patients developed intraoperative dysglycemia. $31.8 \%$ of all intraoperative BG measurements were detected outside the target range. Diabetes [OR $9.263(95 \% \mathrm{Cl} 2.492,34.433)$; $P=0.001]$ and duration of surgery [OR $1.005(1.000,1.010) ; p=0.036]$ were independently associated with the development of intraoperative dysglycemia. Patients who experienced intraoperative dysglycemia had significantly elevated postoperative mean $(p<0.001)$ and maximum BG levels $(p=0.001)$. Length of ICU $(p=0.007)$ as well as hospital stay $(p=0.012)$ were longer in patients with dysglycemia.

CONCLUSIONS: Diabetes and duration of surgery were confirmed as independent risk factors for intraoperative dysglycemia, which was associated with adverse outcome. These patients, therefore, might require intensified glycemic control. Increased awareness and management of intraoperative dysglycemia is warranted.
\end{abstract}

Key words: Intraoperative dysglycemia, hyperglycemia and hypoglycemia, diabetes, risk factors, time-in-target range

\section{Introduction}

Perioperative dysglycemia is frequently seen and associated with postoperative morbidity and mortality [1-3]. Impaired immune function, increased risk of postoperative infections, acute kidney injury, delayed wound healing, postoperative delirium and impaired cognition are recognized as adverse outcomes related to intraoperative hyperglycemia [3-10]. Hypoglycemia is known to contribute to poor postoperative outcome including cardiac arrhythmias, postoperative delirium, increased ICU 
stay and overall mortality, as well [11-13]. Acute insulin resistance is seen as a major cause of intraoperative hyperglycemia due to the surgical stress response enhancing the release of catecholamines and cortisol [14, 15], which can result in so-called stress hyperglycemia [16]. Associated increased glucose variability induces oxidative stress and may also negatively impact patients' recovery [17, 18]. Intraoperative hypoglycemia frequently occurs in response to intensified insulin therapy (IIT). Accordingly, IIT protocols during surgery have produced different results on clinical outcome: While Gandhi et al. [19] and Saager et al. [20] found increased incidences of stroke and delirium; Kwon et al. [21] demonstrated reduced infection and mortality rates.

In fact, evidence suggests that glucose variability resulting in both hyper- and hypoglycemia rather than either hyper- and hypoglycemia alone is critical to clinical outcome, at least in ICU patients $[22,23]$. Targeting glucose levels reduced morbidity and mortality from sepsis in critically ill patients as reported by van den Berghe et al. [24]. Despite the recognition of adverse outcome associated with dysglycemia, understanding of underlying causes of intraoperative dysglycemia is limited. In this subgroup investigation of the BIOCOG study, we aimed to identify risk factors contributing to intraoperative dysglycemia to describe potential causes for glycemic derangement during surgery.

\section{Materials and methods}

\section{Study population}

This subgroup investigation of the Biomarker Development for Postoperative Cognitive Impairment in the Elderly (BIOCOG) study (ethics EA2/092/14, clinicaltrials.gov: NCT02265263, www.biocog.eu) aimed to investigate risk factors of intraoperative dysglycemia in non-cardiac elderly patients. The BIOCOG study is a prospective multicenter observational study to develop biomarkers for risk prediction of postoperative cognitive impairment by assessment of parameters such as clinical, molecular and imaging data [25]. Patients were eligible if at least 65 years of age and if anticipated time of surgery was $\geq 60 \mathrm{~min}$. Patients with a preexisting diagnosis of diabetes as well as non-diabetic patients were included. For the purpose of this subgroup investigation, a subgroup ("SugarCog") of 89 patients enrolled at the site Charite - Universitätsmedizin Berlin between February and December 2016 obtained tight intraoperative blood glucose (BG) measurements every $20 \mathrm{~min}$. These patients were preoperatively planned to receive an arterial catheter for clinical reasons such as anticipated high blood loss or need for continuous monitoring of blood pressure. All patients received guideline-based anesthesiological and surgical treatment according to our standard operating procedures (SOP) [26].

The objective of the study was to identify risk factors for intraoperative dysglycemia. In addition, we investigated the association of intraoperative and postoperative dysglycemia, the association of intraoperative dysglycemia on postoperative outcome, and whether time-out-of-target-range rather than maximum levels are associated with postoperative mortality.

\section{Measurement of intraoperative blood glucose levels}

BG levels were intraoperatively measured immediately after establishment of an arterial catheter after anesthesia induction and then regularly every 20 min until extubation. BG levels measured until up to 1 hour after extubation were considered as intraoperative data. If the patient was not extubated immediately after surgery, BG levels were measured until the patient was transferred to the ICU. All arterial blood gas analyses (ABG) were performed by ABL800 FLEX Radiometer. Dysglycemia was defined according to our SOP as BG levels outside the recommended target range of $80-150 \mathrm{mg} / \mathrm{dL}$ [26]. Patients were subsequently categorized into normoglycemia (80-150 mg/dL) and dysglycemia (at least one intraoperative measurement outside the target range). Minimum, mean and maximum BG levels were determined. Glycemic variability was calculated as the standard deviation of all available intraoperative measurements. Additionally, we determined minutes out of target range from begin until end of surgery.

\section{Determination of postoperative blood glucose levels}

Postoperative BG levels were abstracted from patients' electronic ICU charts. We included ICU measurements only as BG was assessed at regular time intervals as opposed to non-ICU-patients where BG assessment was performed infrequently and at irregular intervals. The same criteria as for intraoperative dysglycemia were applied to all postoperative BG measurements.

\section{Perioperative inflammatory markers}

C-reactive protein (CRP) levels obtained within clinical routine were abstracted from patients' charts. CRP values preoperatively and on postoperative day 1 were compared to investigate a relationship between the inflammatory state and dysglycemia. 


\section{Clinical outcome parameters}

Postoperative complications were documented up to hospital discharge and retrospectively classified according to the Clavien-Dindo classification [27]. For further analyses, we considered Clavien-Dindo grade I-II as minor and grade $\geq \mathrm{III}$ as major complications [28]. Length of ICU stay, time until hospital discharge and 90-day mortality were obtained.

\section{Statistical analysis}

Results are expressed as median (25\%; $75 \%$ quartiles), or $\mathrm{n}$ frequencies (\%), respectively. Differences between groups were tested using non-parametric exact Mann-Whitney-U test for continuous parameters and exact Chi-Square-test for qualitative data. To quantify surgical time-out-of-target range, we calculated the ratio of time outside the target range from begin until end of surgery. Multivariable logistic regression analysis was performed to identify factors associated with intraoperative dysglycemia. Age, sex, American Society of Anesthesiologists (ASA) status (II versus $\geq \mathrm{III}$ ), diabetes, type (intra-abdominal/-thoracic versus other) and duration of surgery, minimum intraoperative hemoglobin $(\mathrm{Hb})$ as well as mean rate of norepinephrine over anesthesia time were entered into the final model. A detailed list of types of surgeries depicts Supplement Table S1. Odds ratios (OR) with 95\%-confidence intervals (CI) and the corresponding $\mathrm{p}$-values were calculated for each risk factor. In a post-hoc approach, the most predictive time point for dysglycemia to occur was determined by a receiver operating characteristics (ROC) curve. Between-group differences regarding postoperative glycemic profiles and clinical outcome were assessed by univariate testing, i.e. exact non-parametric Mann-Whitney-U test and exact Chi-Square-test, respectively. In addition, using the exact Mann-Whitney-U test, maximum BG levels and time-out-of-target range were compared between survivors and non-survivors 90 days after surgery, in order to test for differences in intraoperative dysglycemia.

For the purpose of this exploratory subgroup investigation, no a priori sample size calculation was performed. All results have to be understood in the area of purely exploratory data analysis. No adjustments for multiple testing have been made and p-values do not allow for confirmatory generalization of results. A two-tailed p-value $<0.05$ was considered statistically significant. Analyses were carried out using IBM@ SPSSC Statistics (version 25) and The R Project for Statistical Computing (version 3.4.1).

\section{Ethics}

Ethical approval was obtained (Ethikkommission der Charité - Universitätsmedizin Berlin, EA2/092/14). All patients gave written informed consent upon enrolment. The study complied with the Declaration of Helsinki.

\section{Results}

\section{Study population}

Of 89 patients included in this subgroup investigation, 87 had a prospectively investigated tight intraoperative BG measurement and were, thus, included in the final analysis (Figure 1). Of these, 58 also had at least one postoperative BG measurement by clinical staff due to admission to the ICU. All patients received general anesthesia. One patient required mechanical ventilation due to hypothermia after the end of surgery and was extubated in the ICU 5 hours later. All other patients were extubated immediately after end of surgery. 1354 BG levels were intraoperatively measured $[15(9 ; 21)$ per patient]. $31.8 \%$ of all measured BG levels were outside the target range of $80-150 \mathrm{mg} / \mathrm{dL}$ (Figure 2). $1309 \mathrm{BG}$ levels were postoperatively obtained $[13(6 ; 28)$ per patient]. Over all patients, intraoperative minimum and maximum BG was $64 \mathrm{mg} / \mathrm{dL}$ and $325 \mathrm{mg} / \mathrm{dL}$, respectively. Postoperatively, minimum and maximum BG was $53 \mathrm{mg} / \mathrm{dL}$ and $357 \mathrm{mg} / \mathrm{dL}$, respectively.

Intraoperative dysglycemia occurred in 46 out of 87 patients $(52.9 \%)$. Table 1 demonstrates the distribution of patient baseline characteristics, intraand postoperative variables between patients with intraoperative dysglycemia and those within target range. Patients with dysglycemia had significantly more often a primary diagnosis of diabetes mellitus and had a longer duration of surgery.

\section{Risk factors for intraoperative dysglycemia}

Adjusting for age, sex, ASA status, diabetes, type and duration of surgery, minimum $\mathrm{Hb}$ as well as intraoperative mean rate of norepinephrine over anesthesia time, multivariable logistic regression revealed only diabetes and duration of surgery as independently associated with dysglycemia (Table 2). In a post-hoc approach, plotting of the ROC curve revealed a surgical time of $134 \mathrm{~min}$ most predictive for dysglycemia to occur (sensitivity $71.7 \%$, specificity $63.4 \%$, AUC 0.678, $p=0.004$ ). Course of intraoperative BG levels between diabetic and non-diabetic patients is shown in Figure 3. 
Patients with intraoperative dysglycemia showed increased postoperative maximum glucose levels

High incidences of postoperative dysglycemia were seen in both groups (Table 3). Patients with intraoperative dysglycemia showed increased postoperative maximum $(\mathrm{p}=0.001)$ and mean BG levels $(\mathrm{p}<0.001)$. Postoperative dysglycemia occurred in 18 patients $(46.2 \%)$ who experienced intraoperative dysglycemia but had no previous diagnosis of diabetes.

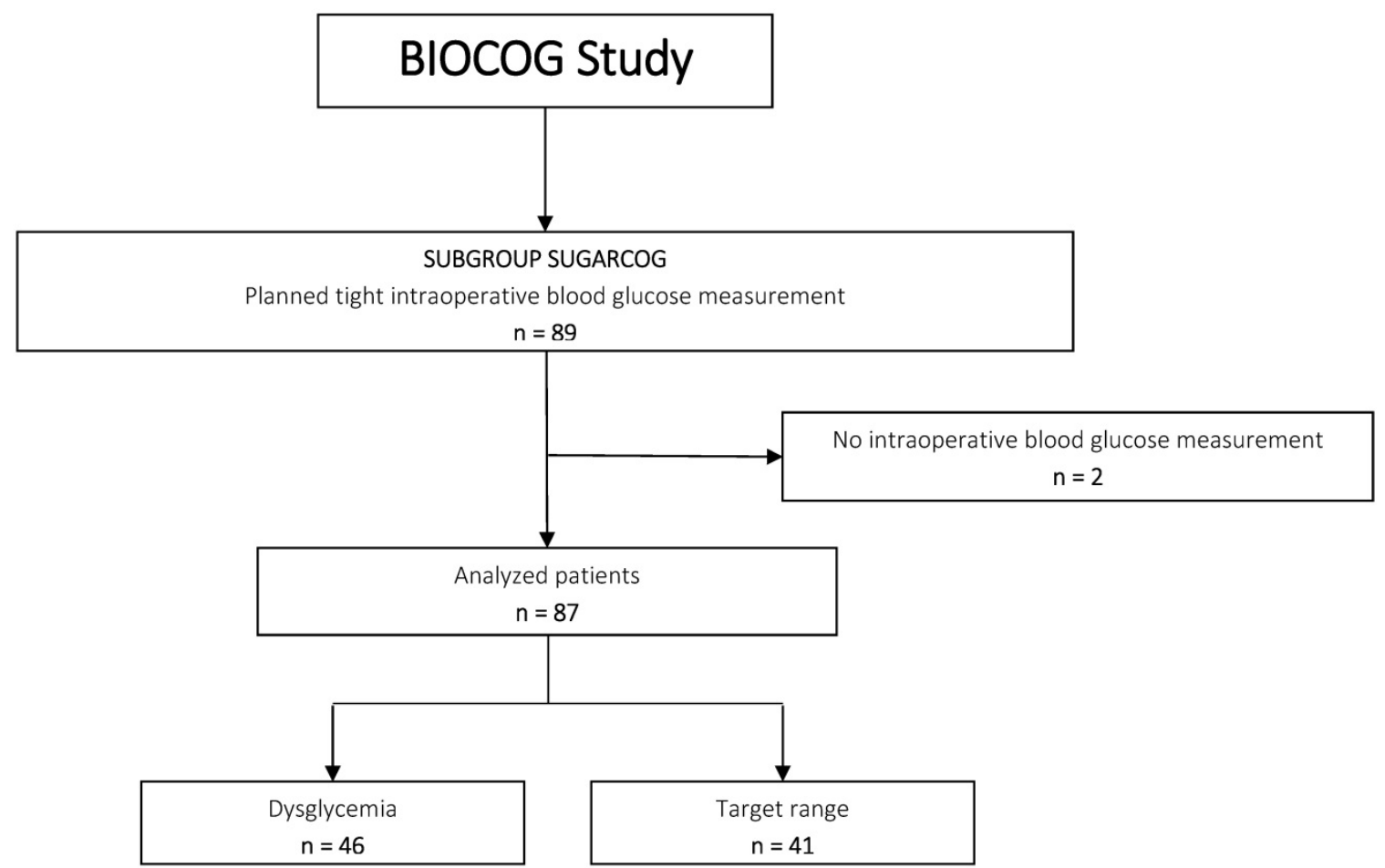

Figure 1.-Consort diagram.

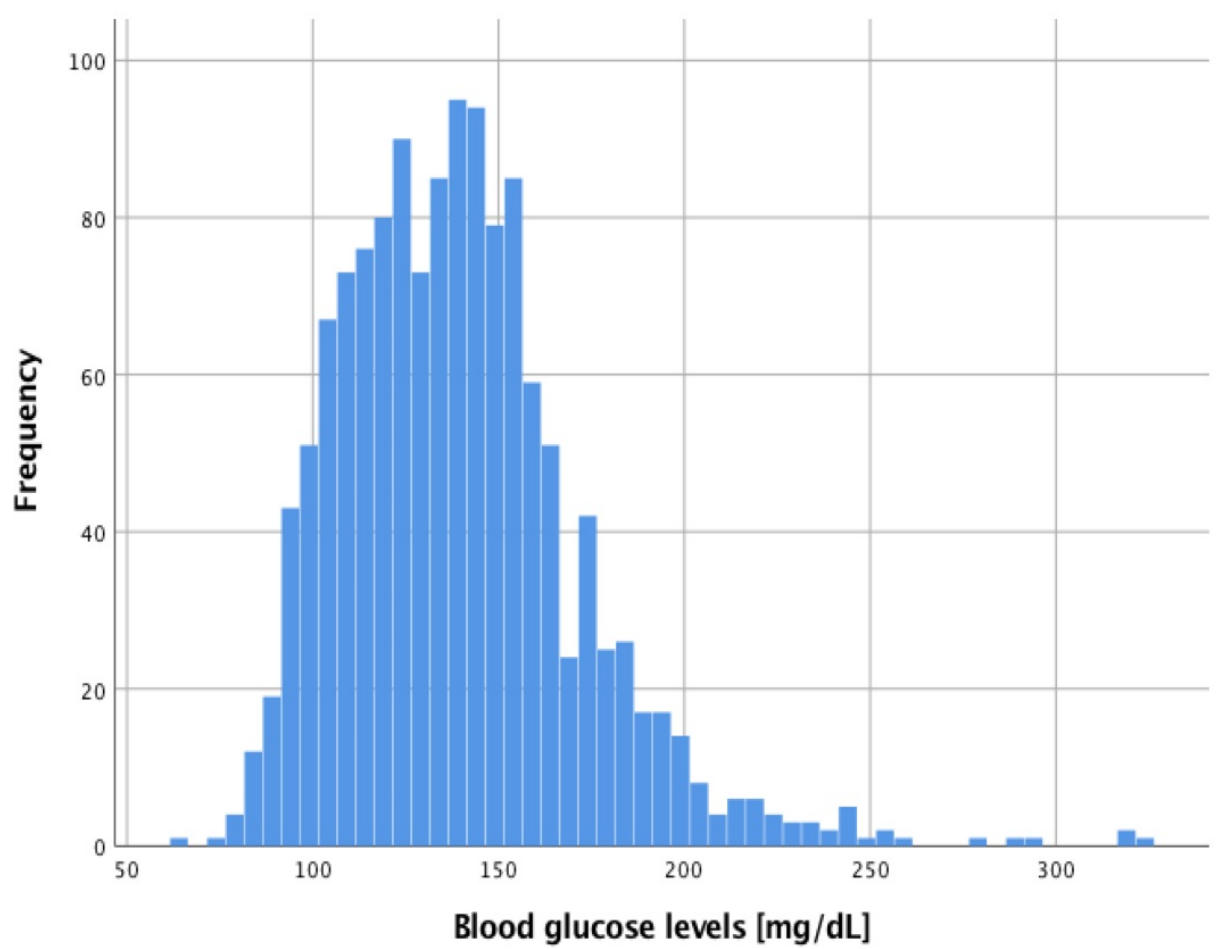

Figure 2.- Histogram of all 1354 intraoperative blood glucose measurements. 


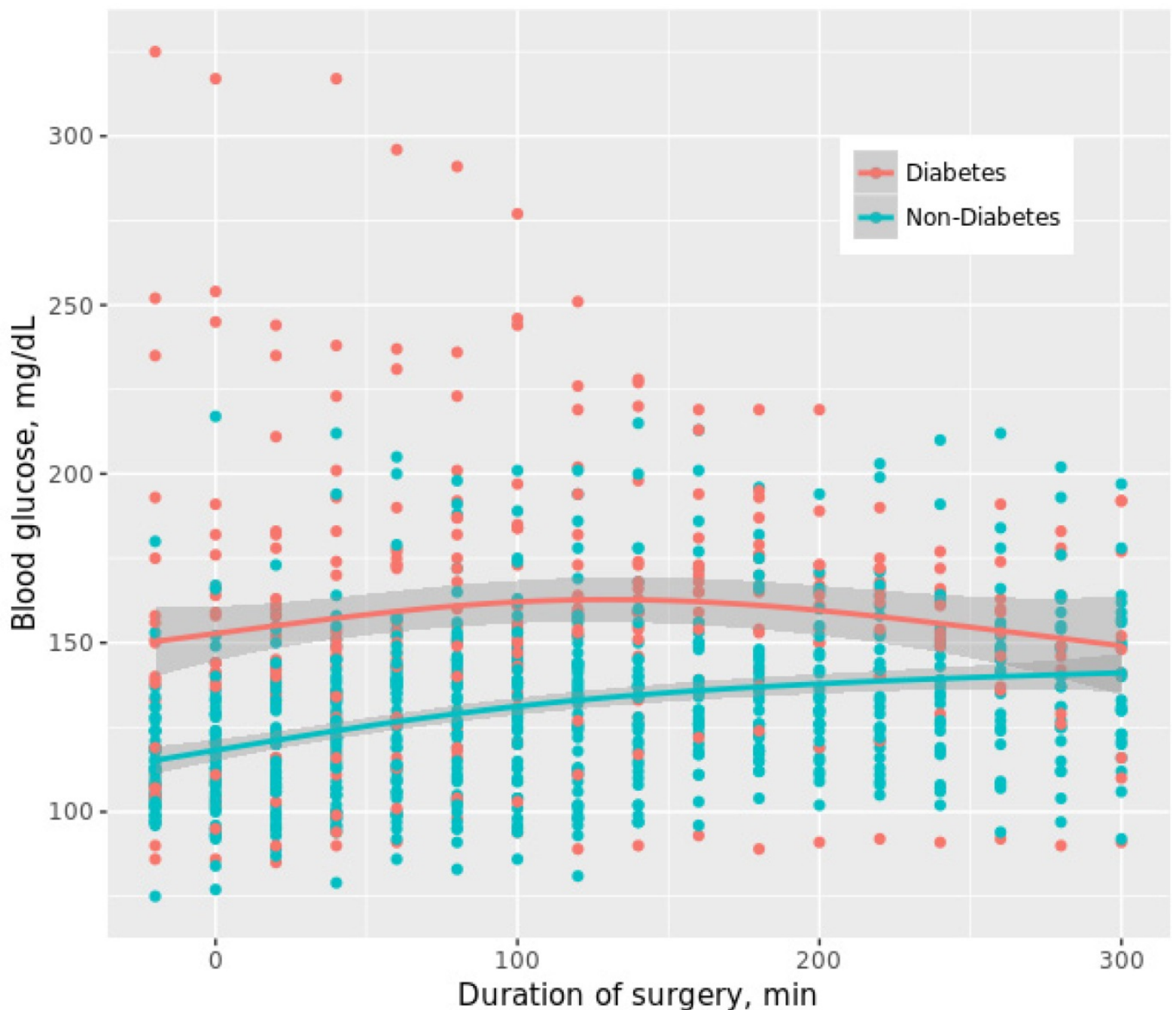

Figure 3. Time course of intraoperative blood glucose levels between diabetic and non-diabetic patients. Eleven out of 27 (40.7\%) diabetic patients received intraoperative insulin (median $8 \mathrm{IU}(4 ; 18)$ ) while 7 out of $60(11.7 \%)$ non-diabetic patients were treated with intraoperative insulin (median $12 \mathrm{IU}(4 ; 14)$ ). For reasons of comparability between diabetic and non-diabetic patients, measurements exceeding $300 \mathrm{~min}$ are not shown to maintain $>5$ measurements per group. First time point was measured immediately after anesthesia induction when the arterial catheter was established. Plotted curves indicate median BG values; grey bands reflect $95 \%$ confidence intervals of the respective group.

Table 1.Study population and characteristics.

\begin{tabular}{|c|c|c|c|c|}
\hline & Total Sample $(n=87)$ & Dysglycemia $(n=46)$ & Target range $(\mathrm{n}=41)$ & $\mathrm{p}$ value \\
\hline \multicolumn{5}{|l|}{ Baseline Characteristics } \\
\hline Age, years & $74(69 ; 77)$ & $72(69 ; 23)$ & $75(71 ; 78)$ & $0.286^{*}$ \\
\hline Sex, male n (\%) & $42(48.3 \%)$ & $20(43.5 \%)$ & $22(53.7 \%)$ & $0.394 \dagger$ \\
\hline $\mathrm{BMI}, \mathrm{kg} / \mathrm{m}^{2}$ & $27(23 ; 30)$ & $26(23 ; 29)$ & $27(23 ; 30)$ & $0.971^{*}$ \\
\hline ASA status, $\mathrm{n}(\%)$ & & & & $0.522 \dagger$ \\
\hline ASA II & $43(49.4 \%)$ & $21(45.7 \%)$ & $22(53.7 \%)$ & \\
\hline ASA III & $44(50.6 \%)$ & $25(54.3 \%)$ & $19(46.3 \%)$ & \\
\hline Charlson Comorbidity Index & $2(2 ; 3)$ & $3(2 ; 4)$ & $2(2 ; 3)$ & $0.163^{*}$ \\
\hline Malignancy, n (\%) & $61(70.1 \%)$ & $34(73.9 \%)$ & $27(65.9 \%)$ & $0.485 \dagger$ \\
\hline Diabetes mellitus, $\mathrm{n}(\%)$ & $27(31.0 \%)$ & $21(45.7 \%)$ & $6(14.6 \%)$ & $0.002 \dagger$ \\
\hline Preoperative $\mathrm{HbA1c}$, \% & $5.7(5.2 ; 6.0)$ & $5.9(5.2 ; 6.8)$ & $5.5(5.2 ; 5.9)$ & $0.025^{*}$ \\
\hline Insulin, $\mathrm{n}(\%)$ & $18(20.7 \%)$ & $17(37.0 \%)$ & $1(2.4 \%)$ & $<0.001 \dagger$ \\
\hline Metformin, n (\%) & $8(9.2 \%)$ & $5(10.9 \%)$ & $3(7.3 \%)$ & $0.717 \dagger$ \\
\hline Steroids, $\mathrm{n}(\%)$ & $5(5.7 \%)$ & $2(4.3 \%)$ & $3(7.3 \%)$ & $0.663 \dagger$ \\
\hline CRP & $5.7(2.7 ; 20.8)$ & $8.4(3.0 ; 23.3)$ & $5.1(2.3 ; 17.2)$ & $0.577^{*}$ \\
\hline ADL & $100(95 ; 100)$ & $100(95 ; 100)$ & $100(95 ; 100)$ & $0.985^{*}$ \\
\hline IADL & $8(8 ; 8)$ & $8(8 ; 8)$ & $8(8 ; 8)$ & $0.368^{*}$ \\
\hline \multicolumn{5}{|l|}{ Intraoperative Characteristics } \\
\hline Type of surgery & & & & $0.056 \dagger$ \\
\hline Intra-abdominal/-thoracic, $\mathrm{n}(\%)$ & $70(80.5 \%)$ & $41(89.1 \%)$ & $29(70.7 \%)$ & \\
\hline
\end{tabular}




\begin{tabular}{|c|c|c|c|c|}
\hline Other, n (\%) & $17(19.5 \%)$ & $5(10.9 \%)$ & $12(29.3 \%)$ & \\
\hline Duration of surgery, min & $233(131 ; 360)$ & $280(178 ; 401)$ & $166(120 ; 303)$ & $0.004^{*}$ \\
\hline Glucose $<80 \mathrm{mg} / \mathrm{dL}, \mathrm{n}(\%)$ & $2(2.3 \%)$ & $2(4.3 \%)$ & 0 & $0.496 \dagger$ \\
\hline Glucose $>150 \mathrm{mg} / \mathrm{dL}, \mathrm{n}(\%)$ & $45(51.7 \%)$ & $45(97.8 \%)$ & 0 & $<0.001 \dagger$ \\
\hline Glucose out of target range, minutes & $0(0 ; 132)$ & $118(65 ; 244)$ & 0 & $<0.001^{*}$ \\
\hline Proportion (\%) of time-out-of-target range & $0(0 ; 66)$ & $60(26 ; 91)$ & 0 & $<0.001^{*}$ \\
\hline Glucose minimum, mg/dL & $109(96 ; 125)$ & $124(103 ; 140)$ & $102(93 ; 113)$ & $<0.001^{*}$ \\
\hline Glucose mean, mg/dL & $131(117 ; 184)$ & $152(138 ; 172)$ & $117(108 ; 127)$ & $<0.001^{*}$ \\
\hline Glucose maximum, mg/dL & $153(133 ; 184)$ & $182(158 ; 206)$ & $133(119 ; 144)$ & $<0.001^{*}$ \\
\hline Glucose variability, mg/dL & $13(7 ; 18)$ & $16(13 ; 23)$ & $8(5 ; 12)$ & $<0.001^{*}$ \\
\hline Insulin, $\mathrm{n}(\%)$ & $18(20.7 \%)$ & $17(37.0 \%)$ & $1(2.4 \%)$ & $<0.001 \dagger$ \\
\hline Insulin, IU & $10(4 ; 15)$ & $10(4 ; 13)$ & $30(30 ; 30)$ & $0.111^{*}$ \\
\hline PRBC, $\mathrm{mL}$ & $0(0 ; 310)$ & $0(0 ; 620)$ & $0(0 ; 155)$ & $0.092 *$ \\
\hline $\mathrm{FFP}, \mathrm{mL}$ & $0(0 ; 1320)$ & $110(0 ; 1980)$ & $0(0 ; 330)$ & $0.007^{*}$ \\
\hline $\mathrm{Hb}$ minimum, g/dL & $9.3(8.1 ; 11.4)$ & $9.1(7.9 ; 10.9)$ & $9.6(8.5 ; 11.9)$ & $0.110^{*}$ \\
\hline Norepinephrine mean rate over anesthesia time, $\mu \mathrm{g} / \mathrm{kg} / \mathrm{min}$ & $0.07(0.03 ; 0.09)$ & $0.07(0.03 ; 0.10)$ & $0.05(0.03 ; 0.07)$ & $0.029^{*}$ \\
\hline Epidural catheter, n (\%) & $45(51.7 \%)$ & $27(58.7 \%)$ & $18(43.9 \%)$ & $0.201 \dagger$ \\
\hline \multicolumn{5}{|l|}{ Postoperative Characteristics } \\
\hline CRP POD1 & $86.9(42.9 ; 118.5)$ & $75.3(43.5 ; 140.9)$ & $88.7(40.8 ; 100.8)$ & $0.885^{*}$ \\
\hline Clavien-Dindo, n (\%) & & & & $0.123 \dagger$ \\
\hline Minor & $48(55.2 \%)$ & $23(50.0 \%)$ & $25(61.0 \%)$ & \\
\hline Major & $35(40.2 \%)$ & $23(50.0 \%)$ & $12(29.3 \%)$ & \\
\hline LOS ICU, h & $23(0 ; 110)$ & $45(18 ; 211)$ & $20(0 ; 46)$ & $0.007^{*}$ \\
\hline LOS, d & $9(6 ; 20)$ & $14(8 ; 25)$ & $8(5 ; 13)$ & $0.012^{*}$ \\
\hline 90d mortality, n (\%) & $16(18.4 \%)$ & $10(21.7 \%)$ & $6(14.6 \%)$ & $0.422 \dagger$ \\
\hline
\end{tabular}

Data are shown as median with quartiles $(25 \% ; 75 \%)$ or as frequencies n (\%). P values are calculated using the exact Chi Square test respectively.

ADL: Activities of Daily Living; ASA: American Society of Anesthesiologists; BMI: Body Mass Index, CCI: Charlson Comorbidity Index; CRP: C-reactive protein; FFP: Fresh Frozen Plasma; Hb: Hemoglobin; IADL: Instrumental Activities of Daily Living; ICU: Intensive Care Unit; LOS: Length of Stay; POD: Postoperative day; PRBC: Packed red blood cells

Table 2. Risk factors for intraoperative dysglycemia.

\begin{tabular}{lll}
\hline Predictors & Odds Ratio $(95 \% \mathrm{CI})$ & p value \\
\hline Age & $0.974(0.883,1.075)$ & 0.600 \\
Sex (male) & $0.511(0.174,1.503)$ & 0.223 \\
ASA status & $1.599(0.513,4.979)$ & 0.418 \\
Diabetes & $9.263(2.492,34.433)$ & 0.001 \\
Intra-abdominal/-thoracic surgery & $4.863(0.926,25.532)$ & 0.062 \\
Duration of surgery & $1.005(1.000,1.010)$ & 0.036 \\
Hb, minimum & $1.187(0.821,1.716)$ & 0.363 \\
Norepinephrine, mean rate & $118767.902(0.072$, & 0.110 \\
( $\mu$ g/ kg/min) & $196869515621.882)$ & \\
\hline
\end{tabular}

ASA: American Society of Anesthesiologists; Hb min: Hemoglobin, minimum intraoperative concentration

Multivariate logistic regression with intraoperative dysglycemia as response was performed with age, sex, ASA status, diabetes, type and duration of surgery, minimum hemoglobin and intraoperative mean rate of norepinephrine over anesthesia time as covariates.

Table 3. Postoperative glycemic profile between patients with and without intraoperative dysglycemia.

\begin{tabular}{llll}
\hline & $\begin{array}{l}\text { Dysglycemia } \\
(\mathrm{n}=39)\end{array}$ & $\begin{array}{l}\text { Target range } \\
(\mathrm{n}=19)\end{array}$ & $\mathrm{p}$ value \\
\hline Glucose minimum, mg/dL & $102(88 ; 126)$ & $93(83 ; 105)$ & $0.117^{*}$ \\
Glucose mean, mg/dL & $142(131 ; 165)$ & $123(115 ; 136)$ & $<0.001^{*}$ \\
Glucose maximum, mg/dL & $198(170 ; 225)$ & $165(147 ; 180)$ & $0.001^{*}$ \\
Postoperative dysglycemia, $\mathrm{n}(\%)$ & $34(87.2 \%)$ & $14(73.7 \%)$ & $0.270 \dagger$ \\
Diabetes, $\mathrm{n}(\%)$ & $17(43.6 \%)$ & $1(5.3 \%)$ & $0.003^{*}$ \\
HbA1c $(\%)$ & $5.8(5.2 ; 6.7)$ & $5.2(5.1 ; 6.0)$ & $0.085^{*}$ \\
\hline
\end{tabular}

Data are shown as median with quartiles $(25 \% ; 75 \%)$ or as frequencies $\mathrm{n}(\%)$. P values are calculated using the exact Chi Square test $\dagger$ and the exact

Mann-Whitney-U test*, respectively.

Intraoperative dysglycemia was associated with adverse clinical outcome

No differences were seen in terms of postoperative complications with regard to
Clavien-Dindo classification (Table 1). However, dysglycemic patients had a significantly longer ICU $(p=0.007)$ as well as hospital LOS $(p=0.012)$. When differentiating between patients with and without preexisting diabetes, those who experienced dysglycemia without diabetes $(n=25)$ showed a trend towards higher mortality rates compared to patients with both diabetes and intraoperative dysglycemia $(n=21)$ [deceased $8(32.0 \%)$ versus $2(9.5 \%) ; p=0.084)$ ].

\section{Discussion}

This study is the first to investigate risk factors for the development of intraoperative dysglycemia in a mixed surgical cohort. The primary finding of this study is that diabetes and duration of surgery were associated with intraoperative dysglycemia independent of age, sex, ASA status, type of surgery, minimum $\mathrm{Hb}$ and mean norepinephrine rate over anesthesia time. A surgical time of at least $134 \mathrm{~min}$ was most predictive for developing intraoperative dysglycemia. Furthermore, dysglycemic patients were at higher risk for postoperative hyperglycemia and adverse postoperative outcome. Importantly, only two patients experienced hypoglycemia whereas hyperglycemia was detected in 45 patients including one with both hypo- and hyperglycemia. Consequently, hyper- rather than hypoglycemia challenges intraoperative BG management. However, both require attention as hypoglycemia might ensue from overtreatment of hyperglycemia.

To the moment, extent rather than duration of surgery has been considered a major contributor to 
perioperative hyperglycemia [10]. Clarke et al. [29] found major surgery to be associated with more severe dysglycemia reasoning that extended surgical trauma leads to increased inflammation and subsequently to a more pronounced stress response. However, our results indicate that duration rather than the extent of surgery contributes to dysglycemia, suggesting that surgical time is critical to progress of the inflammatory response. Subsequently, insulin resistance in response to surgical stress and its related inflammatory process might be aggravated over time, thereby underscoring the key role of time in the development of dysglycemic states.

Even though intraoperative dysglycemia likely occurred in response to inflammatory stress, no difference between patients with dysglycemia and those without was seen in CRP levels neither preoperatively nor 1 day after surgery. As demonstrated by Thorell et al. [30], cytokine release, as one component of the surgical stress response, is related to reduced insulin sensitivity. The authors reported a relationship between increased IL-6 plasma concentrations and a decrease in insulin sensitivity. However, those findings were limited to the postoperative period. Yet, others detected an IL-6 increase early during surgery suggesting that IL-6 mediated insulin resistance increases BG levels over time [31]. This holds important implications for intraoperative BG management. Strategies to control the inflammatory stress response might be considered in order to reduce stress hyperglycemia. Although no difference with regard to dysglycemia was seen in our cohort between patients with and without epidural anesthesia, its use has been demonstrated to reduce the neuroendocrine stress response [32]. An effect similar to that of dexmedetomidine was shown by Shamim et al. [33]. They reported an attenuated stress response in patients receiving dexmedetomidine during laparascopic pyeloplasty. Patients treated with dexmedetomidine had significantly lower levels of serum cortisol and BG. Besides measures to prevent intraoperative hyperglycemia, monitoring its development is of particular concern. Given that the development of dysglycemia is related to surgical time, intensified glycemic control is justified with increasing length of surgery. Anesthesiologists should be aware of the increased risk for dysglycemia suggesting intensified BG monitoring, at least in increased surgical time. Detecting dysglycemia at an early time point allows for adequate therapy. Since dysglycemia is an adverse event for which treatment is easily available, i.e. administering of either insulin or glucose, prevention by thorough monitoring should be the objective of intraoperative BG management.
In our analysis, the critical time for dysglycemia to occur was after 134 minutes. Of note, $31.8 \%$ of all BG measurements lay outside the target range. Although BG was monitored closely in this cohort and appropriate therapy was available, glucose concentrations outside the target range remained high. These findings point out an underestimation of intraoperative dysglycemia. Therefore, close BG measurement needs to be included into clinical routine while dysglycemia should be treated immediately.

Patients with a known diagnosis of diabetes were at higher risk to develop intraoperative dysglycemia. It is acknowledged that diabetic patients demonstrate higher BG levels intraoperatively [34]. Yet, Masla et al. [35] found no difference in intraoperative BG levels between diabetic and non-diabetic patients undergoing coronary artery bypass grafting (CABG). However, this might be due to the fact that the authors assessed BG every 30 minutes only during bypass time. Alterations in BG during on-pump CABG might differ from those occurring during non-cardiac surgery.

Diabetic patients are less likely to experience adverse postoperative outcome compared to non-diabetic patients with dysglycemia $[2,36]$. This might reflect adaption to higher BG levels in that diabetic patients can cope inflammatory and oxidative stress [2,37]. In consequence, different BG target ranges might be recommended for patients with diabetes while patients without diabetes benefit from early intervention to prevent damage from acute hyperglycemia [37]. The fact that intraoperative dysglycemia was related to elevated BG maximum levels in the postoperative period indicates that intraoperatively acquired imbalances influence metabolic homeostasis after surgery. These findings suggest that intraoperative stress hyperglycemia might persist in the postoperative period also in non-diabetic patients. Nair et al. [38] reported similar findings showing that intraoperative elevated mean glucose levels were associated with elevated postoperative levels. Consequently, it can be assumed that intraoperatively dysglycemic patients are at high risk to develop hyperglycemia postoperatively. The recognition that dysglycemia developing intraoperatively is related to postoperative hyperglycemia which itself is a predictor for increased morbidity and mortality, warrants preventive strategies for dysglycemia. Furthermore, in patients with dysglycemia, tight glycemic control should be maintained during the postoperative period to avoid adverse outcomes [21, 39]. In future studies, it will also be of interest to investigate whether a history of dysglycemia related to surgery in non-diabetic 
patients is associated with an increased risk to develop diabetes mellitus.

In the clinical context of surgical ICU patients, it has been acknowledged that targeting BG levels within a prespecified range improves clinical outcome $[24,40]$. However, data on the impact of intraoperative glycemic control and the association of intraoperative and postoperative dysglycemia in non-cardiac patients remain scarce [38]. Gandhi et al. identified intraoperative hyperglycemia as a predictor for adverse outcome including increased mortality after cardiac surgery [41]. A trial conducted by the same research group investigating the influence of intraoperative insulin therapy revealed no reduction in postoperative morbidity but rather an increase of death and stroke related to tight glycemic control, which was attributed to hypoglycemia after insulin therapy [19]. These findings contrast those of Bláha et al. [34] who reported a reduction in postoperative complications when comparing intraoperative tight glucose control to postoperative initiation supporting the concept of glucose target control.

In our study population of 87 patients, dysglycemia was seen in $46(52.9 \%)$ patients of which $21(45.7 \%)$ had a known diagnosis of diabetes indicating that in more than $50 \%$ of all cases, dysglycemia developed in patients without a previous diagnosis of diabetes, which is well described as stress hyperglycemia [16]. In previous studies, differences occurred in patients with diabetes and those without, although both experience an increase in glucose levels in response to surgical stress [42]. However, stress hyperglycemia in non-diabetics leads to a relatively higher rise in BG levels affecting coagulation and immune function and increasing the risk for adverse cardiovascular events [5, 43, 44]. Consequently, in the critically ill without known diabetes, hyperglycemia has deleterious effects on outcome including survival, whereas patients with diabetes seem to be less affected [45, 46]. This is consistent with our finding: Non-diabetics with dysglycemia showed increased 90-day mortality. These protective effects of chronic hyperglycemia on acute hyperglycemia have been attributed to a downregulation of glucose transporters protecting tissues from glucose toxicity [37]. Langouche et al. demonstrated reduced inflammatory activation of the endothelium as a result of insulin therapy providing a rationale for insulin administration to maintain euglycemia [47]. Yet, complete understanding of the pathophysiology of stress hyperglycemia requires further investigation.

Since dysglycemia can be diagnosed during surgery, and should, thus, be preventable if recognized in time, a concept of a standardized protocol for intraoperative glycemic control is warranted. However, in order to target intraoperative BG levels, the underlying factors of dysglycemia need to be identified. We hereby hope to contribute to a better understanding of the development of dysglycemia.

This study has obvious limitations. First, our sample size of 87 patients aged $\geq 65$ years limits generalizability to different patient groups. Second, we did not assess insulin resistance nor was continuous glucose monitoring available. Particularly insulin resistance as underlying pathophysiology might be of great interest in further studies. Third, total blood loss was not assessed and could not be adjusted for in regression analysis. Given that blood loss may also indicate surgical stress, BG levels could also have been influenced. Fourth, no marker indicating intraoperative inflammation was available to support the pathobiology of prolonged inflammation leading to the development of dysglycemia. Only CRP measurements were obtained pre- and postoperatively which are insufficient to assess the inflammatory response in the perioperative period. Finally, survival data were only obtained until day 90 after surgery limiting long-term follow-up.

\section{Conclusions}

The duration of surgery and preexisting diabetes were confirmed as independent risk factors for the occurrence of intraoperative dysglycemia, while age, sex, ASA status, type of surgery, minimum $\mathrm{Hb}$ and norepinephrine had no influence. These patients were also at risk for severe postoperative hyperglycemia and adverse outcome. Further studies are warranted to identify preventive strategies such as tight glycemic control.

\section{Supplementary Material}

Supplementary tables.

http://www.medsci.org/v16p0665s1.pdf

\section{Acknowledgements}

The research leading to these results has received funding from the European Union Seventh Framework Program [FP7/2007-2013] under grant agreement $n^{\circ}$ 602461. Gunnar Lachmann and Tobias Wollersheim are participants of the Clinician Scientist Program granted by the Berlin Institute of Health (BIH). We acknowledge support from the German Research Foundation (DFG) and the Open Access Publication Fund of the Charité - Universitätsmedizin Berlin.

\section{Authors' contributions}

Conceived and designed the experiments: TW, 
CS, GL. Performed the experiments: CK, TW, RM, VW, SK, MK, GL. Analysed the data: CK, GV, VW, GL. Wrote the manuscript: CK, VW, GL.

\section{Competing Interests}

The authors have declared that no competing interest exists.

\section{References}

1. Ascione R, Rogers CA, Rajakaruna C, Angelini GD. Inadequate blood glucose control is associated with in-hospital mortality and morbidity in diabetic and nondiabetic patients undergoing cardiac surgery. Circulation. 2008; 118: 113-23.

2. Frisch A, Chandra P, Smiley D, Peng L, Rizzo M, Gatcliffe C, et al. Prevalence and clinical outcome of hyperglycemia in the perioperative period in noncardiac surgery. Diabetes care. 2010; 33: 1783-8.

3. Shanks AM, Woodrum DT, Kumar SS, Campbell DA, Jr., Kheterpal S. Intraoperative hyperglycemia is independently associated with infectious complications after non-cardiac surgery. BMC Anesthesiol. 2018; 18: 90.

4. Turina M, Miller FN, Tucker CF, Polk HC. Short-term hyperglycemia in surgical patients and a study of related cellular mechanisms. Ann Surg. 2006; 243: 845-51; discussion 51-3.

5. Lachmann G, von Haefen C, Wollersheim T, Spies C. Severe perioperative hyperglycemia attenuates postoperative monocytic function, basophil count and $\mathrm{T}$ cell activation. Minerva anestesiologica. 2017; 83: 921-9.

6. Song JW, Shim JK, Yoo KJ, Oh SY, Kwak YL. Impact of intraoperative hyperglycaemia on renal dysfunction after off-pump coronary artery bypass. Interactive cardiovascular and thoracic surgery. 2013; 17: 473-8.

7. Hermanides J, Qeva E, Preckel B, Bilotta F. Perioperative hyperglycemia and neurocognitive outcome after surgery: a systematic review. Minerva anestesiologica. 2018; 84: 1178-88.

8. Zhang X, Yan X, Gorman J, Hoffman SN, Zhang L, Boscarino JA. Perioperative hyperglycemia is associated with postoperative neurocognitive disorders after cardiac surgery. Neuropsychiatric disease and treatment. 2014; 10: 361-70.

9. Pasternak JJ, McGregor DG, Schroeder DR, Lanier WL, Shi Q, Hindman $\mathrm{BJ}$, et al. Hyperglycemia in patients undergoing cerebral aneurysm surgery: its association with long-term gross neurologic and neuropsychological function. Mayo Clinic proceedings. 2008; 83: 406-17.

10. Duggan EW, Carlson K, Umpierrez GE. Perioperative Hyperglycemia Management: An Update. Anesthesiology. 2017; 126: 547-60.

11. Inouye SK, Westendorp RG, Saczynski JS. Delirium in elderly people. Lancet. 2014; 383: 911-22.

12. Kalra S, Bajwa SJ, Baruah M, Sehgal V. Hypoglycaemia in anesthesiology practice: Diagnostic, preventive, and management strategies. Saudi J Anaesth. 2013; 7: 447-52.

13. Investigators N-SS, Finfer S, Chittock DR, Su SY, Blair D, Foster D, et al. Intensive versus conventional glucose control in critically ill patients. The New England journal of medicine. 2009; 360: 1283-97.

14. Thorell A, Nygren J, Ljungqvist O. Insulin resistance: a marker of surgical stress. Current opinion in clinical nutrition and metabolic care. 1999; 2: 69-78.

15. Barth E, Albuszies G, Baumgart K, Matejovic M, Wachter U, Vogt J, et al. Glucose metabolism and catecholamines. Critical care medicine. 2007; 35: S508-18.

16. Palermo NE, Gianchandani RY, McDonnell ME, Alexanian SM. Stress Hyperglycemia During Surgery and Anesthesia: Pathogenesis and Clinical Implications. Current diabetes reports. 2016; 16: 33.

17. Esposito K, Nappo F, Marfella R, Giugliano G, Giugliano F, Ciotola M, et al. Inflammatory cytokine concentrations are acutely increased by hyperglycemia in humans: role of oxidative stress. Circulation. 2002; 106: 2067-72.

18. Monnier L, Mas E, Ginet C, Michel F, Villon L, Cristol JP, et al. Activation of oxidative stress by acute glucose fluctuations compared with sustained chronic hyperglycemia in patients with type 2 diabetes. Jama. 2006; 295: 1681-7.

19. Gandhi GY, Nuttall GA, Abel MD, Mullany CJ, Schaff HV, O'Brien PC, et al. Intensive intraoperative insulin therapy versus conventional glucose management during cardiac surgery: a randomized trial. Ann Intern Med. 2007; 146: 233-43.

20. Saager L, Duncan AE, Yared JP, Hesler BD, You J, Deogaonkar A, et al. Intraoperative tight glucose control using hyperinsulinemic normoglycemia increases delirium after cardiac surgery. Anesthesiology. 2015; 122: 1214-23.

21. Kwon S, Thompson R, Dellinger P, Yanez D, Farrohki E, Flum D. Importance of perioperative glycemic control in general surgery: a report from the Surgical Care and Outcomes Assessment Program. Ann Surg. 2013; 257: 8-14

22. Hermanides J, Vriesendorp TM, Bosman RJ, Zandstra DF, Hoekstra JB, Devries JH. Glucose variability is associated with intensive care unit mortality. Critical care medicine. 2010; 38: 838-42.

23. Kauffmann RM, Hayes RM, Buske BD, Norris PR, Campion TR, Jr., Dortch $\mathrm{M}$, et al. Increasing blood glucose variability heralds hypoglycemia in the critically ill. The Journal of surgical research. 2011; 170: $257-64$

24. van den Berghe G, Wouters P, Weekers F, Verwaest C, Bruyninckx F, Schetz $\mathrm{M}$, et al. Intensive insulin therapy in critically ill patients. The New England journal of medicine. 2001; 345: 1359-67.

25. Winterer G, Androsova G, Bender O, Boraschi D, Borchers F, Dschietzig $\mathrm{TB}$, et al. Personalized risk prediction of postoperative cognitive impairment - rationale for the EU-funded BioCog project. European psychiatry : the journal of the Association of European Psychiatrists. 2018; 50: 34-9.

26. Spies C, Kastrup M, Kerner T, Melzer-Gartzke C, Zielke H, Kox WJ. SOPs in der Anästhesiologie und Schmerztherapie. Stuttgart: Georg Thieme Verlag KG; 2013

27. Dindo D, Demartines N, Clavien PA. Classification of surgical complications: a new proposal with evaluation in a cohort of 6336 patients and results of a survey. Ann Surg. 2004; 240: 205-13.

28. Casadei R, Ricci C, Pezzilli R, Calculli L, D'Ambra M, Taffurelli G, et al. Assessment of complications according to the Clavien-Dindo classification after distal pancreatectomy. JOP : Journal of the pancreas. 2011; 12: 126-30.

29. Clarke RS. The hyperglycaemic response to different types of surgery and anaesthesia. Br J Anaesth. 1970; 42: 45-53.

30. Thorell A, Loftenius A, Andersson B, Ljungqvist O. Postoperative insulin resistance and circulating concentrations of stress hormones and cytokines. Clinical nutrition. 1996; 15: 75-9.

31. Cruickshank AM, Fraser WD, Burns HJ, Van Damme J, Shenkin A. Response of serum interleukin- 6 in patients undergoing elective surgery of varying severity. Clinical science. 1990; 79: 161-5.

32. Ahlers O, Nachtigall I, Lenze J, Goldmann A, Schulte E, Hohne C, et al. Intraoperative thoracic epidural anaesthesia attenuates stress-induced immunosuppression in patients undergoing major abdominal surgery. Br J Anaesth. 2008; 101: 781-7.

33. Shamim R, Srivastava S, Rastogi A, Kishore K, Srivastava A. Effect of Two Different Doses of Dexmedetomidine on Stress Response in Laparoscopic Pyeloplasty: A Randomized Prospective Controlled Study. Anesth Essays Res. 2017; 11: 1030-4.

34. Blaha J, Mraz M, Kopecky P, Stritesky M, Lips M, Matias M, et al. Perioperative Tight Glucose Control Reduces Postoperative Adverse Events in Nondiabetic Cardiac Surgery Patients. The Journal of clinical endocrinology and metabolism. 2015; 100: 3081-9.

35. Masla M, Gottschalk A, Durieux ME, Groves DS. HbA1c and diabetes predict perioperative hyperglycemia and glycemic variability in on-pump coronary artery bypass graft patients. Journal of cardiothoracic and vascular anesthesia. 2011; 25: 799-803.

36. Puskas F, Grocott HP, White WD, Mathew JP, Newman MF, Bar-Yosef S. Intraoperative hyperglycemia and cognitive decline after CABG. The Annals of thoracic surgery. 2007; 84: 1467-73.

37. Dungan KM, Braithwaite SS, Preiser JC. Stress hyperglycaemia. Lancet. 2009; 373: 1798-807.

38. Nair BG, Horibe M, Neradilek MB, Newman SF, Peterson GN. The Effect of Intraoperative Blood Glucose Management on Postoperative Blood Glucose Levels in Noncardiac Surgery Patients. Anesthesia and analgesia. 2016; 122: 893-902.

39. Mohan S, Kaoutzanis C, Welch KB, Vandewarker JF, Winter S, Krapohl $\mathrm{G}$, et al. Postoperative hyperglycemia and adverse outcomes in patients undergoing colorectal surgery: results from the Michigan surgical quality collaborative database. International journal of colorectal disease. 2015; 30: 1515-23.

40. Griesdale DE, de Souza RJ, van Dam RM, Heyland DK, Cook DJ, Malhotra A, et al. Intensive insulin therapy and mortality among critically ill patients: a meta-analysis including NICE-SUGAR study data. CMAJ : Canadian Medical Association journal $=$ journal de l'Association medicale canadienne. 2009; 180: 821-7.

41. Gandhi GY, Nuttall GA, Abel MD, Mullany CJ, Schaff HV, Williams BA, et al. Intraoperative hyperglycemia and perioperative outcomes in cardiac surgery patients. Mayo Clinic proceedings Mayo Clinic. 2005; 80: $862-6$. 
42. Vanhorebeek I, Van den Berghe G. Diabetes of injury: novel insights. Endocrinology and metabolism clinics of North America. 2006; 35: 859-72, $x$.

43. Lefebvre PJ, Scheen AJ. The postprandial state and risk of cardiovascular disease. Diabetic medicine : a journal of the British Diabetic Association. 1998; 15 Suppl 4: S63-8.

44. Coutinho M, Gerstein HC, Wang Y, Yusuf S. The relationship between glucose and incident cardiovascular events. A metaregression analysis of published data from 20 studies of 95,783 individuals followed for 12.4 years. Diabetes care. 1999; 22: 233-40.

45. Rady MY, Johnson DJ, Patel BM, Larson JS, Helmers RA. Influence of individual characteristics on outcome of glycemic control in intensive care unit patients with or without diabetes mellitus. Mayo Clinic proceedings. 2005; 80: 1558-67.

46. Egi M, Bellomo R, Stachowski E, French CJ, Hart GK, Hegarty C, et al. Blood glucose concentration and outcome of critical illness: the impact of diabetes. Critical care medicine. 2008; 36: 2249-55.

47. Langouche L, Vanhorebeek I, Vlasselaers D, Vander Perre S, Wouters PI, Skogstrand K, et al. Intensive insulin therapy protects the endothelium of critically ill patients. The Journal of clinical investigation. 2005; 115: 2277-86. 